\title{
Virtual worlds as art practice: EvoArt methodologies
}

\author{
Rui Filipe Antunes \\ Goldsmiths, University of London \\ rui.antunes@gold.ac.uk
}

\author{
Frederic Fol Leymarie \\ Goldsmiths, University of London \\ ffl@gold.ac.uk
}

\begin{abstract}
Synthetic biology as an artistic technique draws on the exploration of the mechanisms used by organisms to process information and develop their morphologies to address a diverse range of aesthetic agendas. The instrumentalisation of biological processes and methodologies by artists is called Evolutionary Art (EvoArt) and this practice has been attracting in the last three decades an increasing number of creators. Natural selection and genetics are inspirations for the practice of artists such as William Latham (development of biomorphic shapes: sculptures, paintings, designs), or Robb Lovell and John Mitchell (development of entire virtual ecologies).
\end{abstract}

As Virtual Worlds (VWs) establish themselves at the centre of the technical and social panorama of the internet, they become more determinant in the contextualisation of human communication and sociability, and at the same time establish themselves as metaphors of cultural processes for the contemporary human condition. In the present advent of VWs, one of the main challenges that EvoArt artists face is precisely extending their knowledge and practice into this new technological and cultural realm. In this communication we will identify three EvoArt approaches that can inform VWs construction and aesthetics: (i) living non-player characters, (ii) living architectures, and (iii) collaborative construction of the landscape.

Evolutionary Art, Virtual Worlds, ALife, Visual art, New Media.

\section{INTRODUCTION}

Synthetic biology as an artistic technique draws on the exploration of the mechanisms used by organisms to process information and to develop their morphologies to address a wide range of aesthetic agendas. The instrumentalisation of biological processes and methodologies by artists is called EvoArt and this practice has been attracting in the last three decades an increasing number of authors such as Todd-Latham (Todd \& Lattam, 1989) or McCormack (McCormack, 2004).

Since at least the late 19th century, a growing number of artists, such as Antoni Gaudi, Jean Arp, Henry Moore or Constantin Brancusi, have pursued their formal aesthetic investigations inspired by nature. More recently, the EvoArt artists, working with software, appropriate biological and ecological mechanisms and properties, using them as tools in their artistic practices. Despite distinct outcomes these practices share a similar project. The largest difference is situated in the representational realm, which is now focused on the systemic processes of nature rather than the exterior aspects of reality.

Whitelaw categorises three main dominant methodologies in this artistic genre using software as a medium and Artificial Life as technique: breedings, cybernatures and abstract works (Whitelaw, 2004). Whitelaw refers to the artists as breeders who employ genetic strategies to produce visuals, such as Latham and Todd's FormGrowth; cybernatures encompass the sub-genre of works involving the creation of ecologies in silico, such as Christa Sommerer's A-Volve; IMA Traveller from Driessens \& Verstappen provides an example of abstract works.

Major investments realised in recent years on virtual worlds (VW) and the adoption of developed platforms by the mainstream culture are together challenging our perception of the internet 'spacescape' and consequently providing new spaces and new forms for artistic expression.

One of the challenges that EvoArt artists face is extending their knowledge and practices into the domain of VWs. Our main hypothesis is that VWs, as spaces simultaneously (bio-) mimetic and fantastic, as well as representational and potentially abstract, can be populated by autonomous entities that can assemble, replicate using blueprints, to which mutations can occur, and that can adapt and interact with the environment in a process that can be observed as an artificial evolution of ecologies. 
These combined properties operate not only on the metaphoric and symbolic realms as they also structure generative outcomes where emergent processes create visual and sublime novelty.

In the next sections, we will first present a brief genealogy of VWs. We will identify three EvoArt approaches that can inform VWs. Then we will explain why VWs are significant contemporary aesthetic instruments, and, finally, we will describe a VW we initiated where some of these identified techniques have been experimentally employed.

\section{A SUMMARISED VIEW OF VIRTUAL WORLDS}

The end of last century witnessed the cultural mainstream consciousness being pervaded by convergent forms of technology. A single technological platform congregates previous electronic media (networking, real-time 3D graphics, audio, video, animation, etc.). The mobile technologies and the Web 2.0 are symptomatic examples of this phenomenon. VWs don't diverge from this general tendency and multimedia is now part of their substratum. Based on this transdisciplinarity, VW are becoming increasingly sophisticated and encompass now a range of services including commercial, social, gaming, and web-browsing functionalities.

The audience, through their avatars, which are graphical representations of themselves, can explore, play and interact, engaging in rich interactions within the world. The idea of being 'inthere' is omnipresent and acts as a common denominator amongst the different sub-genres of VWs (Multi-user social environments, MMORPG and we argue single-user virtual gardens).

\subsection{What is considered as a virtual world?}

Ralph Shroeder et al. have put forward a definition of VW as 'persistent virtual environments in which people experience others as being there with them and where they can interact together (Schroeder et al., 2008). Mark Bell draws on similar lines to suggest VWs are 3D 'environments, persistent, based on computer networks and participated by multi-users mediated by visual representations (Avatars)' (Bell, 2008).

VWs can then be thought of as interactive spaces, where the user can enter an artificial world, become a character in it and create behaviours and relationships with other users in the context of this created environment. This 'definition' is flexible enough to include online gaming and Massively multiplayer online role-playing games (MMORPGs), as usually a line is drawn separating these formats from social spaces based on their activity revolving around gaming and whose central aim is accumulating points or reaching new (game) levels (Schroeder, 2008). In that sense, these online gaming environments are a subset of VWs (Steinkuehler \& Williams, 2006); and extending this reasoning other subsets include some virtual communities with animated representation (which focus primarily on social interaction) and artistic virtual environments such as TechnoSphere (Prophet, 2001) or AVolve (Mignonneau \& Sommerer, 1999).

In the Poetics of Virtual Worlds Lizabeth Klastrup proposes a different definition of VW more inclusive than Bell's and Schroeder's: 'A virtual world is a persistent online representation, which contains the possibility of synchronous interactions between users and between user and world within the framework of a space designed as a navigable universe' (Klastrup, 2003). This definition includes the textual forms of Multi-User Dungeons (MUDs), which are amongst the seminal contributions for VWs.

These various definitions of VWs have their emphasis on a concept of a believable 'out-there' and on the participation and interaction of multiple users. However, no matter how inclusive they are, these definitions still exclude single-user virtual environments where users are able to co-exist and interact with virtual populations of life forms (aka virtual ecosystems).

We explore in the following the adoption in VWs of the generative and evolutionary techniques developed in some single-user virtual environments, in particular the techniques that artists make use in EvoArt.

\subsection{A condensed summary of art practice in VWs}

As cultural objects, artists make use of videogames and VWs to convey their messages. Artists engage in critical debate by questioning videogames as cultural artefacts, such as the Velvet-Strike project, lead by Anne-Marie Schleiner, where a guerrilla group make graffiti on the polygonal walls of the shooter MMO Counter-Strike with pixelated pacifist messages; or using the video environment to address issues from global politics and society such as Wafaa Bilal's hacked version of the game Quest for Saddam, where the artist impersonates a suicide bomber sent on a mission to assassinate the American president Bush. If hacking the game is an artistic gesture by itself, other examples include the design of complete games, such as Escape from Woomera, to identify the audience with the daily life of a detainee on a detention centre for illegal immigrants in Australia.

Laurence Weiner's Homeport is one of the earliest examples of using VWs as spaces for artistic activity. In the VW The Palace, in the 1990s, he 
extended the white cube of Leo Castelli's gallery, representing it covered with symbols and writings. Second Life (SL) in this respect is a major stage for experiments. Similar to Escape from Woomera's first person experience, Nonny de La Pena developed Gome Gitmu, a SL island where she attempts to convey to the audience the experience of a detainee in Guantanamo, which can be interpreted as an artistic/political work. Three main types of artistic approaches in SL are identified by John Lester et al.: (a) visual artists such as 'Not possible in real life', who, operating on a wide scope of themes and techniques (landscape, fashion, architecture) (Lester, 2009), make use of the virtual to go further than the physical limitations of reality and create interactive experiences where the audience can step in and interact; (b) artists like Don Coyote Antonelli and the ZeroG SkyDancers, who use a virtual environment for performative acts, involving live actors, dancers and music represented by avatars, but once again, creating experiences that go further than the limits enabled by reality, such as a stage that rises lifting the audience in Don Coyote's performance, while the dancers fly around the set which changes dynamically based on their motions (Lester, 2009); and (c) artists such as Jeffrey Lipsky making a hybrid use of the virtual and the physical world which are co-permeated in mixed reality installations, where visitors at a physical gallery interact with a virtual gallery and vice versa (Lester, 2009).

These artistic approaches sustain themselves from the power of VWs (such as SL) to induce an idea of worldness to the environment. However, this worldness is not solely sustained on a basis of mimesis. Another quality which is overtly present in VW is the fantastic understood here as the desire to transcend reality. The ZeroG Sky Dancers, despite using humanoid avatars, are not restricted by the laws of physics of our day to day reality, and can, for example, dance in the skies of the world. The fantastic is an integrant part of this world, a strangeness, which can be associated to a dream space rather than common reality.

\section{LIVING VIRTUAL WORLDS}

As we have seen, despite the inclusiveness of Klastrup's definition, previous attempts at clarifying what a VW is exclude an important branch of virtual environments: private gardens in local computers inhabited by virtual populations.

\subsection{Digital aquariums of life}

Synthetic life and ecosystems have already a significant history in Artificial Intelligence (Al). Such Cybernatures, as Whitelaw calls these, are part of a sub-field of Al called ALife which draws from natural (Darwinian) evolution and what we understand of morphogenesis for the development of these virtual populations residing on the memory of computers. Virtual populations inhabit virtual environments and evolve according to their interactions both with other individuals and their habitats. In the typical implementation rudimentary metabolisms force individuals to forage for energy and move and explore the space of the simulations. Individuals are able to reproduce, typically in sexual reproductions, generating new offspring. A process of Darwinian natural selection adapts the gene-pool of the population evolving the genes of the fittest individuals who perform better at finding energy and at reproducing. Complex social behaviours emerge from these worlds, such as mimetism, parasitism, mutualism, etc. Examples of these types of worlds include Thomas Ray's Tierra (Ray, 1990), Larry Yager's Polyworld (Yager, 1994), and Jeffrey Ventrella's Darwin Pond (Ventrella, 2005). Currently in development the EvoGrid aims to develop a robust simulation of the emergence of life forms in a primordial soup making use of a grid of computers (Damer, 2009). With biota.org efforts are situated on developing a set of standards for the interoperability of virtual creatures from different simulations, so that, for instance, creatures from Pond can easily migrate to Polyworld (Damer \& Barbalet, 1995).

\subsection{Evolutionary art in VWs}

VWs are more than social communities and commercial platforms. As the term 'world' implies, they can overcome the reductive view of interactive spaces for avatars. As it stands, they have a humanistic and anthropomorphic view, an inherent divide between animate and inanimate: the avatars and the rest of the world.

EvoArtists are in a privileged position to change this situation. Due to their technical and critical knowhow they can promote a new vision for VWs. Evolution theories and principles have been under scrutiny of EvoArtists. They have appropriated A-life techniques in their practice to develop artistic work inspired by biology and nature. Natural Selection and Genetics are inspirations for artists such as Todd \& Latham (development of biomorphic shapes) or Lovell \& Mitchell (development of entire ecologies). Withelaw (in Metacrations) scrutinises artistic works using this methodology. Jon McCormack's Eden (McCormack, 2001) and Christa Sommerer and Laurence Mignonneau's Life Spacies I and II (Mignonneau and Sommerer, 1997) are examples extending the work on Cybernatures and creating artistic aquariums of digital life.

\subsubsection{The first proposal --- Liquid architectures}

Re-presenting ideas from Marcos Novak's liquid architectures (Novak, 1991), our first proposal is to define VWs as spaces where buildings and other architectures should have autonomous behaviours, and react to their environment and adapt; eventually 
even reproduce and evolve. John Frazer drew on similar lines of thought and using A-life techniques conceived of space as an ecology of dynamically drawn objects (Frazer, 1995). Don Coyote's performances explore some of these facets in a clear demonstration of its validity in the new territory of multi-user VWs.

\subsubsection{The second proposal --- Evolutive NPCs}

On the other hand, not only can architectures be different in VWs (than in reality), but they can also incorporate living 'Non Player Characters' (NPCs). NPCs, also called autonomous characters, are creatures that populate video game worlds; generally they have some form of limited 'intelligence' and have some ability to improve their actions (Reynolds, 1999).

Our second proposition is that VWs should be ecologies populated with genomic-based creatures that evolve according to their interactions with their environment and neighbours. Implicit in this proposal, the system's dynamics need to handle principles of natural evolution.

\subsubsection{The third proposal --- Collaboration}

Our final proposal is to provide VWs with the potential for collaborative construction of the landscape. Scott Draves showed how the participation from multiple users could provide rich visual outcomes. In the Electric Sheep project, users share the unused computing power of their own machines to render imagery from scripts previously sent from a central server. Once rendered, the different participants send their computed outputs to the main server that assembles them together and produces a collective work (Draves, 2007). VWs can build on this experience to create collaborative elements of the landscape, such as the backgrounds.

In summary, we are elaborating series of virtual universes of living landscapes, architectures, and creatures, with active interactions from users.

\section{CONSIDERATIONS ON THE VW AS AESTHETIC INSTRUMENT}

Considering the technological substratum of VWs, it can be suggested that VWs exist in an ontological dichotomy. If on the one hand, ultimately, they are code and data residing in computer memories, and streams of such code/data being transferred from clients to servers in a network of computers, on the other hand, they are based conceptually on the idea of 'worldness'.

\subsection{The VW as generative space}

Approaching VWs from a phenomenological point of view reinstates questions from Software art practice.
Namely the dichotomy promoted by those who understand software as a generative mechanism unreflexive and focused on the processual relations of coding and aesthetic output, a position illustrated by the works of Joshua Davies and Lia; and those who on the other side, for instance Mathew Fuller, understand software as a cultural artefact and use code for its political and sociological implications visualising rhetorical entity/relation structures (Whitelaw, 2005). Mitchell Whitelaw discusses this dichotomy in his paper called System Stories and Model Worlds. Addressing Lev Manovitch's vision of Generative art as a metaphoric illustration of contemporary social complexity, and reverberating the virtual as potential as envisioned by Pierre Lévy, Whitelaw sustains that the extra value of Generative art is the possibility of engaging critically with the systems themselves, to 'tinker...then set them running: possible worlds' (Whitelaw, 2005).

We can then argue that when artists reinstantiate Christofer Langton's Life-as-it-could-be, on the one hand, and make use of 'simulation techniques...as generative devices' (Whitelaw, 2005), they are reformulating these techniques as alternative critical instruments.

\subsection{The code aesthetics}

A second aspect noteworthy about code is that it is not only crucial in terms of the conceptualisation of the work as well as it is determinant and decisive in the visual outcome and impact of the work. In a similar way as different temperatures have influenced different drying times on painting oils in Northern Europe and this enabled the emergence of an aesthetics distinct from Venice and Florence's the code also drives and filters many aesthetic decisions, and consequent visual outcomes. The implementation of the hardware functionalities is different in each language and the palette of functions available differs among them. Building on VRML, or using scripting languages from Second Life, or game engines such as Unity3d is operating on distinct aesthetic domains from using, for example, the repertoire of functions from Java $3 D$ or of direct implementations in Open GL. Artists working in Second Life (SL) are restricting their praxis to the scope of functions provided by the APIs from Linden Labs (the creators of SL), and consequently to operate under Linden Labs' implementation of the hardware features. The choice of the implementation language is consequently an aesthetic and ethical decision.

\subsection{The 'worldness' on the VW: mimesis and metaphor}

Typically VWs have been used as intrinsic spaces of mimesis and representation. Lizbeth Klastrup, drawing on the idea of 'worldness' of the world, 
suggests an understanding of the world "both as a fiction, a social space, a performative space and a special form of game' (Klastrup, 2003). She deconstructs the world in four functions: as an interpretative framework; a prop; a simulation and as a lived narrative. The world as interpretative framework, or fiction, is the concept or story behind the world. It constitutes a reference point from which the actions make sense; for instance the story of its creation and evolution, the cosmology of its inhabitants. The interpretative framework contributes significantly to situate the elements in a common and shared territory, a contextual space and time.

The concepts Paideia and Ludus defined by Roger Caillois (Caillois, 2001) are reinstated in the world as simulation and shared prop. These functions are proximal to play, game and performance space. Combined they provide an understanding of the world as a specific game. As shared prop the audience engages within the world in a makebelieve attitude, embodying an avatar representation or pretending to be someone else. In the function of simulation, the success of the navigation and interaction on the audience in the VW depends on his/her knowledge of rules. These functions appear to be connected as in both situations the audience pretends to be in the world, interacting with the real objects in a game environment.

Finally the function of the world as lived narrative or story producer refers to aspects originated and experienced during the interactions with the world. The world understood as a stage for a shared experience (Klastrup, 2003).

As we have seen before, the 'worldness' of the VW constitutes the basic model of expression used by many artists in commercial VWs such as SL.

\subsection{The VW as a broaden metaphor}

However as artistic medium VWs are not limited to the representational dominion: As we just have described in the previous points VWs incorporate two non-exclusive conceptual fields: the materialphenomenological and the metaphoricrepresentational. The association of the representational realm with the generative dynamics of the processes of computational rendering of the world, this dual quality of the world as system and as metaphor, is what constitutes VWs as exceptional theatres for artistic expression and permit an aesthetical exploration of VWs that is quite distinct and exclusive to this particular medium.

Extrapolating what Holmes conjectures about virtual reality and virtual communities, we can posit that VWs offer metaphors of the broader on-going cultural processes, but are as well beginning to deeply impact means of human communication (Holmes, 1997). Lev Manovitch, suggests that computer software and its interfaces manifest clearly the new aesthetics of information culture. He wrote: 'never before was a single machine an engine of economy and the main tool for representation' (Manovich, 2001). In this respect it is always noteworthy to cite the illustration of the hyperreal condition of the city of Los Angeles captured by Lev Manovitch - in his words: 'a precise model for the virtual world' (Manovich, 1995). If that is the case (and we agree with Manovich), we can quote Domenico Quaranta in this respect when he proposes: 'the challenge before us is to figure out how to employ these tools to create new art' (Quaranta, 2006).

In the next section we will present a work which implements EvoArt techniques and illustrate how living environments can significantly enrich VWs. This model of using EvoArt contrasts with the current paradigm of worldness in VW, which can be described as 'a preset response to user navigation through an ossified database' (Penny, 2004).

\section{AN ECO-VIRTUAL WORLD}

Understanding VWs as interpretative frameworks and contexts for simulation and living narratives, as Lizbeth Klastrup describes them, permits approaching these environments as textual and narrative spaces, which depend on the user's agency to make associations and mental reconstructions in the process of interpretation. Klastrup uses the term 'multi-user textuality' to describe the forms of text resulting from multiple distributed users interacting via network. In her media-inclusive proposition, textuality is described as 'the place of the reader and text in the process of reading. The text contains signs that the reader, in the process of reading, decodes and interprets, be it graphical, auditive or verbal signs'. She continues 'the text does not necessarily need to be written text, but can be all forms of cultural artefacts with a signifying function' (Klastrup, 2002). Extending the notion of 'digital textuality' she widens the notion of textual construction to encompass all the technological elements participating in the interactive experience of the world.

\subsection{Senhora da Graca: VWs as generative spaces of representation}

The VW of Senhora da Graça aims to explore the textual features of Cybernatures to produce a representational artefact: a memorial representing the physical space sharing the same name, which was submerged in 2000 with the rising waters of a newly constructed dam. 


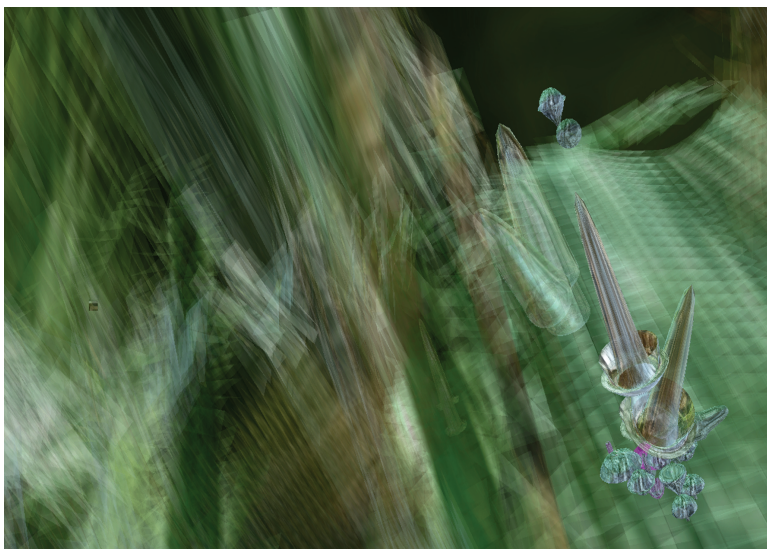

Figure 1: Still image from Senhora da Graça, showing a tree where some herbivores feed

Aesthetic decisions were made about this work taking in consideration the code and the material implementation of the world. The interpretative framework of the world is influenced by its development using Java3D, its polygonal nature and its rendering processes. A narrative is built on top of the $3 \mathrm{D}$ textures and the material evolution and deformation of the polygonal 3D meshes, contributing to its final aesthetics.

The virtual Senhora da Graça is a space of mimesis understood in the sense described by Katherin Hume, as the process of imitation, description of events, people, situations and objects, with a resemblance that enables others to share the experience (Hume, 1995).

Senhora da Graça is a virtual ecosystem composed of clouds of rain, soil, plants, herbivores, carnivores and scavengers. With the help of photographs taken at the site of Senhora da Graça, about 20 years ago, this work was aimed to reference a specific period, an historical moment in time. However, the photographs are presented in a distorted and somewhat abstract way, when applied as 3D textures on the exterior surfaces of the creatures (skin), on the soil, and in the skies of the VW. As the surfaces are not static, the generative dynamics of the interaction of creatures permanently rebuilds the world and the shapes. As a result, the photographs keep making reference to a moment of time but the living and dynamic frames where they are applied (e.g. the creature's bodies) as 3D textures, evolve in time making them unrecognisable. Having lost their pictorial value as photographic object they keep maintaining their conceptual and chromatic values. In a metaphoric way, as it happens with the submerged physical space of the real site of Senhora da Graça, in this work the photographs appear unrecognisable: they are shadows of the historical moment they evoke (Antunes \& Leymarie 2009).

\section{CONCLUSIONS}

A new form of artistic expression is emerging in VWs. The possibilities for artistic expression that the generative, narrative and representative dimensions of VWs present are distinct and characteristic of this medium, creating unique outcomes that cannot be achieved with any other medium.

We have elaborated on the idea of 'interpretative framework', constituted by the aggregate of elements composing the world, to construct the idea of the VW as an artwork, whose materiality is explored artistically first through the aspect of its substratum as code and data; secondly as a generative space where the 'worldness' of the VW, virtual life, and the audience (user) interaction, creates permanent narratives and perpetual novelty. The case study presented, a representational art system in the form of a VW, suggests an enrichment of the ontology of VWs with these novel generative approaches transferred into what we may call EcoEvo-Art.

\section{ACKNOWLEDGMENTS}

This work is supported in part by the Fundação para a Ciência e Desenvolvimento from Portugal, in the form of a PhD studentship for Mr. Antunes.

\section{REFERENCES}

Antunes, R. F. and Leymarie, F. F. (2009) Senhora da Graça, a virtual world as memorial. In Rosa M. P. Oliveira, (ed.) Ciantec 2009 - III congresso internacional das artes, novas tecnologias e comunicação, Aveiro, Portugal.

Bell, M. W. (2008) Toward a Definition of "Virtual Worlds", Journal of Virtual Worlds Research, 1 (Virtual Worlds Research: Past, Present \& Future): p. 1.

Caillois, R. (2001) Man, play and games. Urbana: University of Illinois Press.

Damer B. (2009) Summoning the EvoGrid. Biota live podcast (44), March 29, 2009 online:

http://www.biota.org/podcast/live.html (1 April 2010).

Damer B. and Barbalet T. (1995) Biota.org. online:http://www.biota.org/about/ (1 April 2010).

Draves S. (2007) Evolution and Collective Intelligence of the Electric Sheep. In Romero J. and Machado P. The Art of Artificial Evolution: $A$ Handbook on Evolutionary Art and Music, Springer.

Frazer J. (1995) An Evolutionary Architecture, Themes VII, Architectural Association, London. 
Holmes D. ed. (1997) Virtual politics: identity and community in cyberspace. Sage Publications.

Hume K. (1985) Fantasy and Mimesis: Responses to Reality in Western Literature. Methuen.

Mitchell J. D. and Lovell R. E. (1995) EIDEA: An Emergent Interactive Installation - Environment for the Interactive Design of Emergent Art. In International Symposium on Electronic Art, Montreal, Canada.

Klastrup L. (2002) A virtual world aesthetics: theorising multi-user textuality. Internet Research, online:http://www.itu.dk/people/klastrup/airpapfinalve r.pdf (1 April 2010).

Klastrup L. (2003) A Poetics of Virtual Worlds. in Fifth International Digital Arts and Culture Conference., Melbourne: RMIT. Online: http://hypertext.rmit.edu.au/dac/papers/Klastrup.pdf (1 April 2010)

Lester J. (2009) Artistic Expression in Second Life: What can we learn from creative pioneers of new mediums? In Journal of Virtual Worlds Research, 1 (Cultures of Virtual Worlds)

http://journals.tdl.org/jvwr/article/view/535/432 (1 April 2010)

Manovich L. (1995) Global Algorithm 1.3: The Aesthetics of Virtual Worlds: Report From Los Angeles. http://www. manovich.net/TEXT/virtspace.html (1 April 2010)

Manovich L. (2001) Info-Aesthetics: Information and Form. http://www.manovich.net//A/ (1 April 2010)

McCormack J. (2001) Eden: an evolutionary sonic ecosystem. In J. Kelemen Sosik and P. (ed.), Lecture Notes in Artificial Intelligence, Vol 2159, Advances in Artificial Life, Proc. of the 6th European Conference (ECAL), Prague, Czech Republic, Springer-Verlag, Berlin, Germany, pp. 133-142.

Mignonneau L. and Sommerer C. (1997) Life Spacies, ICC Concept Book, NTT-ICC, Tokyo, pp. 96-101.

Mignonneau L. and Sommerer C. (1999) Art as a Living System: Interactive Computer Artworks. In System, 32(3), pp. 165-173.

Novak M. (1991) Liquid Architectures in Cyberspace, MIT Press, pp. 248-251.

Penny S. (2004) Cited In M. Whitelaw. Metacreation,. MIT Press, p. 194.
Prophet J. (2001) "Real" Time, "Artificial" Life. In Leonardo Vol 34 (4), pp. 309-312.

Quaranta, D. (2006) Game Aesthetics. In M. Bittanti and D. Quaranta (eds.) GameScenes: Art in the Age of Videogames. Johan and Levi, Milan.

Ray T. S. (1990) An approach to the synthesis of life In C. Langton (ed.) Artificial Life II, pp. 371-408. Addison Wesley, Santa Fe, New Mexico,

Reynolds C. (1999) Steering Behaviors for Autonomous Characters In Game Developers Conference, pp. 763-782.

Schroeder R. (2008) Defining Virtual Worlds and Virtual Environments Journal of Virtual Worlds Research, 1 (Virtual Worlds Research: Past, Present \& Future), pp. 1-3. journals.tdl.org/jivwr/article/download/294/248 (1 April 2010)

Steinkuehler, C. and Williams, D. (2006) Where everybody knows your (screen) name: Online games as "third places" Journal of ComputerMediated Communication, Vol 11(4) article 1. http://icmc.indiana.edu/vol11/issue4/steinkuehler.ht $\underline{\mathrm{ml}}$ (1 April 2010)

Todd, S. and Latham W. (1989) Computer Sculpture. IBM Systems Journal, 28(4) pp. 682-688.

Ventrella J. (2005) GenePool: Exploring The Interaction Between Natural Selection and Sexual Selection In A. Adamatzky and M. Komosinski (eds.) Artificial Life Models in Software. Springer London, pp. 81-96.

Whitelaw M. (2004) Metacreation: Art and Artificial Life. MIT Press.

Whitelaw M. (2005) System Stories and Model worlds: A Critical Approach to Generative art. In Readme 100 : Temporary Software Art Factory. pp. 135-154. Books on Demand GmbH.

creative.canberra.edu.au/mitchell/papers/SystemSto ries.pdf (1 April 2010)

Yaeger L. (1994) Computational genetics, physiology, metabolism, neural systems, learning, vision and behavior or polyworld: Life in a new context. In C. G. Langton (ed.) Proceedings of the Artificial Life III Conference, Volume XVII of Santa Fe Institute Studies in the Sciences of Complexity, Addison-Wesley, New York, pp. 263-298. 